\title{
SOME APPLICATIONS OF LÉVY PROCESSES TO STOCHASTIC INVESTMENT MODELS FOR ACTUARIAL USE
}

\author{
BY \\ TERENCE Chan \\ Dept. of Actuarial Mathematics and Statistics \\ Heriot-Watt University
}

\begin{abstract}
This paper presents a continuous time version of a stochastic investment model originally due to Wilkie. The model is constructed via stochastic differential equations. Explicit distributions are obtained in the case where the SDEs are driven by Brownian motion, which is the continuous time analogue of the time series with white noise residuals considered by Wilkie. In addition, the cases where the driving "noise" are stable processes and Gamma processes are considered.
\end{abstract}

\section{KEYWORDS}

Lévy process; Brownian motion; stochastic investment model.

\section{INTRODUCTION}

Wilkie (1986) presented an investment model based on time series, which has since been updated and extended in Wilkie (1995). This paper presents some continuous time variants of Wilkie's original model using stochastic differential equations driven by appropriate Lévy processes. There is no single correct continuous time equivalent to the model in Wilkie (1986); the aim of this paper is to suggest some possible ways of constructing the analogous continuous time models and to analyse these mathematically. It seems that whatever one takes to be the "right" continuous time equivalent of the Wilkie model, similar methods to those presented here can be used to analyse it.

One reason one might be interested in a continuous time model is that in a continuous time setting one is free to choose any unit of time and to model the state of the various investment variables at any time, not just at discrete instants. However, the main attraction of continuous time models is their 
mathematical tractability; whereas the Wilkie model is mainly intended for computer simulations, in the continuous time setting here many questions admit explicit answers which can be obtained in a simple way. Here, we concentrate on obtaining explicit distributions but other questions can undoubtedly be answered.

The model introduced in Wilkie (1986) only makes use of Gaussian (white noise) series; for our model the driving noises are allowed to have other distributions.

\section{DESCRIPTION OF THE MODEL}

In many ways, the model described here is the most direct and obvious continuous-time version of the model in Wilkie (1986), although some modifications are necessitated by the transition to a continuous time scale. We do not make any special claims about its appropriateness to practical situations beyond pointing out its similarity to the original Wilkie model which has by now gained wide acceptance, at least in the world of insurance. The two main guiding principles behind the construction of the continuous time model presented here are firstly the analogy with the corresponding time series and secondly the similarities between certain features of the Wilkie model and other models which feature widely in different areas of financial modelling; occasionally we shall depart from an exact analogy with the time series to emphasise these similarities because the qualitative features common to all these models are of potentially greater interest. Thus, it would be more appropriate to refer to the continuous time model presented here as inspired by the Wilkie model, rather than "the continuous time Wilkie model". The model should be treated as a "first draft" rather than a final version. As with the original Wilkie model, the model here is based on four processes (although these are not exactly the same as the ones in Wilkie (1986)) and we describe each of these in turn.

Let $Z_{1}, Z_{2}, Z_{3}$ and $Z_{4}$ be four independent (not necessarily continuous) processes. Exactly what kind of processes are the $Z_{i}$ will be discussed later.

\subsection{Retail prices index and inflation}

Consider first a retail prices index, $Q_{t} \equiv \exp \left\{P_{t}\right\}$. We use an OrnsteinUhlenbeck type model for the process $P$ :

$$
\begin{aligned}
& d P_{t}=R_{t} d t \\
& d R_{t}=-a_{1} R_{t} d t+\phi(t) d t+\sigma_{1} d Z_{1}(t)
\end{aligned}
$$

where $a_{1}>0, \sigma_{1} \in \mathbb{R}$ and $\phi$ is a (deterministic) positive periodic function with period $h>0$. Here the process $R$ plays the role of the continuous force of inflation. A direct translation of Wilkie's model would have $\phi \equiv$ constant, but in passing to continuous time it may be desirable to take into account the seasonal fluctuations in inflation over a year. The period $h$ here corresponds 
to a year in our units of time (see Remark (ii) below). (To spell things out in a little more detail, supposing $\phi \equiv$ constant, the process $R_{t}$ in (1.1) corresponds to Wilkie's $\nabla \log Q(t)$, the parameter $a_{1}$ corresponds to the parameter Wilkie calls 1 - QA, $\phi$ corresponds to QMU(1 - QA) in Wilkie's original paper and $\sigma_{1}$ plays the role of QSD).

Because (1.1) is a linear equation, it is easy to solve explicitly, whatever our choice of driving noise $Z_{1}$. The general version of Itô's formula for discontinuous semimartingales $X$ states that if $f$ is a continuous function with the necessary derivatives,

$$
\begin{aligned}
f\left(X_{t}, t\right) & -f\left(X_{0}, 0\right)=\int_{0}^{t} \dot{f}\left(X_{s}, s\right) d s+\int_{0}^{t} f^{\prime}\left(X_{s-}, s\right) d X_{s}+\frac{1}{2} \int_{0+}^{t} f^{\prime \prime}\left(X_{s-}, s\right) d[X]_{s} \\
& +\sum_{0<s \leq t}\left(f\left(X_{s}, s\right)-f\left(X_{s-}, s\right)-f^{\prime}\left(X_{s-}, s\right) \Delta X_{s}-\frac{1}{2} f^{\prime \prime}\left(X_{s-}, s\right)\left(\Delta X_{s}\right)^{2}\right)
\end{aligned}
$$

where $\Delta X_{s}=X_{s}-X_{s-}, \dot{f} \equiv \partial f / \partial t, f^{\prime} \equiv \partial f / \partial x$ etc. For this and other aspects of the general theory of stochastic integration with respect to semimartingales, we refer the reader to Protter (1990) and Roger and Williams (1987), which approach the subject in different ways. (Note that $X$ is assumed to be right-continuous and can only have countably many jumps, so the sum above is actually a sum over countably many values of $s$ ). Consider now the case that $f(x, t)=e^{a t} X_{t}$. We have $f^{\prime \prime} \equiv 0$ and $f^{\prime}\left(X_{s-}, s\right) \Delta X_{s}=f\left(X_{s}, s\right)-f\left(X_{s--}, s\right)$, so the terms involving the jumps of $X$ in Itô's formula all vanish. Therefore applying Itô's formula to $e^{a_{1} t} R_{t}$, we obtain an explicit formula for $R_{t}$ :

$$
R_{t}=e^{-a_{1} t} R_{0}+\int_{0}^{t} e^{-a_{1}(t-s)} \phi(s) d s+\int_{0}^{t} \sigma_{1} e^{-a_{1}(t-s)} d Z_{1}(s) .
$$

From (1.2), we can find $P_{t}=P_{0}+\int_{0}^{t} R_{s} d s$ and the resulting double integrals can be handled by interchanging the order of integration (e.g. see Lemma 3.1 in the sequel).

\subsection{Share yield process}

Wilkie (1986) next considers two inter-related processes: an index of share dividends and the dividend yield process. Let $Y_{t}$ denote the share dividend yield. The continuous time analogue of Wilkie's model would be

$$
\begin{aligned}
Y_{t} & =Y_{*} \exp \left\{X_{t}+\zeta R_{t}\right\}, \\
\text { where } \quad d X_{t} & =-a_{2} X_{t} d t+b_{1} d t+\sigma_{2} d Z_{2}(t) .
\end{aligned}
$$


(Here, $Y_{*}=Y_{0} e^{-\left(X_{0}+\zeta R_{0}\right)}$. In the sequel, this notation will be frequently used to denote this kind of "modified initial condition".) Equation (1.3) admits an explicit solution similar to (1.2), namely

$$
X_{t}=X_{0} e^{-a_{2} t}+b_{1}\left(\frac{1-e^{-a_{2} t}}{a_{2}}\right)+\int_{0}^{t} \sigma_{2} e^{-a_{2(t-s)}} d Z_{2}(s) .
$$

\subsection{Share dividend process}

We next turn to the index of share dividends, $D_{t}$. Our model follows Wilkie in using an exponentially discounted "sum of inflation effects":

$$
d\left(\log D_{t}\right)=\left(b_{2}+\beta \lambda \int_{0}^{t} e^{-\lambda s} R_{t-s} d s+\gamma R_{t}\right) d t+\eta_{2} d Z_{2}(t)+\eta_{3} d Z_{3}(t) .
$$

In Wilkie's time series model, the noise has a simultaneous as well as a lagged effect which is captured by moving average in the noise. There is no sensible equivalent in the continuous time context for such a moving average. Another feature of the model (inherited from Wilkie) is the mixing of the driving noises for $Y_{t}$ and $D_{t}$.

The share price $S_{t}$ is related to the dividends and the yield by $S_{t}=D_{t} / Y_{t}$. It is interesting to note that the process $S_{t}$ satisfies an equation of the form

$$
d S_{t}=c_{t} S_{t} d t+S_{t}\left(\delta_{1} d Z_{1}(t)+\delta_{2} d Z_{2}(t)+\delta_{3} d Z_{3}(t)\right),
$$

which has exactly the same form as the ubiquitous geometric Brownian motion model of share prices, except that the coefficient $c_{t}$ here takes a rather complicated form which involves the whole path of the force of inflation $R$ up to time $t$, as well as the usual constant drift terms.

Interchanging the order of integration, it is easy to see that

$$
\lambda \int_{0}^{t} \int_{0}^{s} e^{-\lambda u} R_{s-u} d u d s=\int_{0}^{t}\left(1-e^{-\lambda(t-u)}\right) R_{u} d u
$$

therefore from (1.5) we have

$$
D_{t}=D_{*} \exp \left\{\eta_{2} Z_{2}(t)+\eta_{3} Z_{3}(t)+\beta \int_{0}^{t}\left(1-e^{-\lambda(t-u)}\right) R_{u} d u+\gamma \int_{0}^{t} R_{u} d u+b_{2} t\right\},
$$

where $D_{*}$ is a constant determined by $D_{0}$ and $R_{0}$ in a similar manner to $Y_{*}$ (see Remark (iv) in $\$ 1.5$ below). 


\subsection{Consol yield process}

Finally, we have the yield on consols $C_{t}$,

$$
\begin{aligned}
C_{t} & =\xi \rho \int_{0}^{t} e^{-\rho s} R_{t-s} d s+C_{*} e^{V_{t}}, \\
d V_{t} & =-a_{4} V_{t} d t+\sigma_{4} d Z_{4}(t), \quad V_{0}=v
\end{aligned}
$$

The equation for $V$ in (1.7) admits an explicit solution for the same form as (1.2).

\subsection{General remarks on the model}

(i) We do not claim that the method in (1.1) is the most appropriate way to model seasonal effects in inflation - it is one simple and obvious way to do it without destroying the most attractive features of the Ornstein-Uhlenbeck process but we could equally plausibly let $\sigma_{1}$ be a periodic function as well and we would still be able to obtain an explicit solution as before.

(ii) Some remarks on the time scale of the continuous time processes here and their relationship with their discrete-time counterparts in Wilkie (1986) might be useful. Typically these continuous time processes run at a much faster speed than their discrete-time equivalents: for example, if the unit of time in Wilkie (1986) is years, the unit of time here might be centuries, so that $h=0.01$ would correspond to a year. This is essentially an artifact of the discretization in passing from continuous time to discrete time. If we were to discretize (1.1) in multiples of $h$ using first-order Euler approximation together with the approximation $P_{t}-P_{t-h}=\int_{t-h}^{t} R_{s} d s \approx h R_{t-h}$ and noting that $\phi(t)=\phi(t-h)=\phi=$ constant, we would recover the Wilkie model provided we rescale time by defining $\tilde{R}_{t}:=R_{h t}$. For example, assuming that $Z_{1}$ is Brownian motion for simplicity, the first-order Euler discretization of $(1.1)$ is

$$
R_{t}-R_{t-h}=-a_{1} h R_{t-h}+\phi h+\sigma_{1}\left(Z_{1}(t)-Z_{1}(t-h)\right),
$$

which can be rewritten as

$$
\begin{aligned}
R_{t} & =\left(1-a_{1} h\right) R_{t-h}+\phi h+\sigma_{1} \sqrt{h} W_{t} \\
& =\mu+a\left(R_{t-h}-\mu\right)+\sigma_{1} \sqrt{h} W_{t},
\end{aligned}
$$

where we have put $a=1-a_{1} h, \mu=\phi / a_{1}$ and $W_{t}=\left(Z_{1}(t)-Z_{1}(t-h)\right) / \sqrt{h}$. Note that $W_{h}, W_{2 h}, W_{3 h}, \ldots$ are i.i.d. standard Gaussian random variables. Defining $\tilde{R}_{t}:=R_{h t}$, we obtain from (1.8) the AR(1) time-series model of Wilkie:

$$
\tilde{R}_{t}=\mu+a\left(\tilde{R}_{t-1}-\mu\right)+\tilde{\sigma}_{1} W_{t},
$$


where $\tilde{\sigma}_{1}=\sigma_{1} \sqrt{h}$. The calculations at (2.5) and (2.6) below and the subsequent discussion illustrate this point in greater detail. Observe that the corresponding parameters in (1.1) are rescaled in the appropriate way with this time change: $\tilde{a}_{1}=h a_{1}$ and $\tilde{\sigma}_{1}=\sigma_{1} \sqrt{h}$. Because the parameters are automatically scaled accordingly once a time scale has been chosen, such comparisons with the discrete time-series are usually irrelevant from a practical point of view; in practice, one would choose a suitable time scale and then fit the model to data directly without reference to any discrete-time model and if one wished to do simulation, one would choose a discretization for its numerical efficiency rather than for its consistency with another discrete-time model. The same comment applies to all the other processes discussed above.

(iii) For our choices of $Z_{1}$, the process $R$ will have a stationary distribution. Throughout this paper, we assume that the initial condition $R_{0}$ is some fixed number as in (1.2). However, it is also possible to let $R_{0}$ be a random variable with the stationary distribution, in which case $R$ would be a stationary process. The same can be said of all the other processes which have stationary distributions.

(iv) Because the processes $X$ and $R$ in (1.3) are not spatially homogeneous, the initial values $X_{0}$ and $R_{0}$ cannot be absorbed into $Y_{*}$ and so separate parameters for the initial values are needed. The same applies to the processes $D$ and $C$. Also, Wilkie (1986) has an extra drift term of the form $c d t$ appearing in the equation for $V_{t}$ in (1.7) but we have omitted it here because it is clear from the explicit formula for $V_{t}$ that $c$ can be absorbed into the two parameters $v$ and $C_{*}$, and so serves no additional purpose.

\subsection{Lévy processes}

We are mainly interested in the case where the "noise" processes $Z_{i}$ are symmetric Lévy processes, that is processes with stationary independent increments. ("Symmetric" in this context just means that $Z$ and $-Z$ have the same law.) We end this section by briefly recalling some results about Lévy processes which we shall need in the sequel. Let $Z$ be a (symmetric) Lévy process. Since $Z$ has stationary independent increments, its characteristic function must take the form $\mathbb{E}\left[e^{-i \theta Z_{t}}\right]=e^{-t \ell \psi(\theta)}$ for some function $\psi$, called the Lévy exponent of $Z$. The Lévy-Khintchine formula says that

$$
\psi(\theta)=\frac{\sigma^{2}}{2} \theta^{2}+i a \theta+\int_{\{|x|<1\}}\left(1-e^{-i \theta x}-i \theta x\right) \nu(d x)+\int_{\{|x| \geq 1\}}\left(1-e^{-i \theta x}\right) \nu(d x)
$$


for $a, \sigma \in \mathbb{R}$ (if $Z$ is symmetric, $a=0$ ) and for some $\sigma$-finite measure $\nu$ on $\mathbb{R} \backslash\{0\}$ satisfying $\int \min \left(1, x^{2}\right) \nu(d x)<\infty$. The measure $\nu$ is called the Lévy measure of $Z$. (To put readers on more familiar ground, consider the situation when $\sigma=a=0$ and suppose that the total mass $\lambda$ of $\nu, \lambda=\int_{\mathbb{R}} \nu(d x)$ is finite. Then the Lévy process $Z$ with such a Lévy measure is just an ordinary compount Poisson process which jumps occurring as a Poisson process of rate $\lambda$ and whose jump-size distribution is $\lambda^{-1} \nu(d x)$. In the case that the integral of $\nu$ diverges near $0, Z$ will have infinitely many small jumps in a finite time-interval. At the other extreme, if $\nu \equiv 0$, there are no jumps so we just have Brownian motion and $\psi$ is the same as the exponent for a normal distribution.)

From the Lévy-Khintchine formula we can deduce the exact form $Z$ must take: it turns out that $Z$ must be a linear combination of a Brownian motion (the continuous part) and a pure-jump process independent of the Brownian part. Specifically, let $Q(d t, d x)$ be a Poisson measure on $(0, \infty) \times \mathbb{R}\{0\}$ with expectation measure $d t \times \nu$ (here $d t$ denotes Lebesgue measure), then (assuming $a=0$ in (1.10)) we have the Lévy decomposition

$$
Z_{t}=\sigma B_{t}+J_{t}+A_{t}
$$

where, corresponding to each of the three terms in (1.10) respectively, $B$ is a Brownian motion, $J$ is the pure-jump martingale $J_{t}=\int_{|x|<1} x(Q((0, t], d x)-t \nu(d x))$ and $A$ is the finite-variation jump process $A_{t}=\int_{\{|x|>1\}} x Q((0, t], d x)$. The processes $B, J$ and $A$ are independent. A more detailed treatment can be found in Protter (1990) and Rogers and Williams (1987) also contains a nice direct construction of (1.11). Because of independence, we lose no generality in treating separately the cases where $Z$ is a Brownian motion and where $Z$ is a pure-jump process. We do this in the next two sections.

\section{EXPLiCIT DISTRIBUtions IN THE Brownian CASE}

If the $Z_{i}$ are all Brownian motions, all the processes described in the previous section are either Gaussian processes or exponentials of Gaussian processes. Since in order to specify the law of a Guassian process one only has to specify the mean and the covariance, the results of this section are essentially trivial.

Recall that for a Brownian motion $W, \int_{0}^{t} f(s) d W_{s}=B\left(\int_{0}^{t} f(s)^{2} d s\right)$ where $B$ is some other Brownian motion. Applying this result to (1.2) gives

$$
R_{t}=e^{-a_{1} t} R_{0}+\int_{0}^{t} e^{-a_{1}(t-s)} \phi(s) d s+\sigma_{1} e^{-a_{1} t} B_{1}\left(\frac{e^{2 a_{1} t}-1}{2 a_{1}}\right)
$$


where $B_{1}$ is a Brownian motion. Hence, $R_{t}$ has Gaussian distribution with mean

$$
\mu_{R}(t)=e^{-a_{1} t} R_{0}+\int_{0}^{t} e^{-a_{1}(t-s)} \phi(s) d s
$$

and variance

$$
v_{R}(t)=\sigma_{1}^{2}\left(\frac{1-e^{-2 a_{1} t}}{2 a_{1}}\right) .
$$

(In 2.2a,b) we have used the fact that $B_{t}$ is Gaussian with mean 0 and variance $t$ ). Similar results hold for the other Ornstein-Uhlenbeck type processes $X$ and $V$ introduced in Section 1 .

From (1.6) and (1.7), it is clear that the key to finding the distributions of $D_{t}$ and $C_{t}$ lies in obtaining the distribution of $\int_{0}^{t} f(s) R_{s} d s$ for suitable (deterministic) functions $f$. Since $R$ is a Gaussian process, so is $t \mapsto \int_{0}^{t} f(s) R_{s} d s$ and so all we need to do is work out the mean and variance of $\int_{0}^{r} f(s) R_{s} d s$. The mean is trivial: by interchanging the order of integration it is easy to see that the mean is just $\int_{0}^{t} f(s) \mu_{R}(s) d s$. We now turn to the variance. Since the mean is irrelevant here, the variance is simply given by

$$
\mathbb{E}^{0}\left[\left(\int_{0}^{t} f(s) H_{s} d s\right)^{2}\right]=\mathbb{E}^{0}\left[\int_{0}^{t} \int_{0}^{t} f(s) f(u) H_{s} H_{u} d u d s\right]
$$

where we have put

$$
H_{t}=\sigma_{1} e^{-a_{1} t} B_{1}\left(\frac{e^{2 a_{1} t}-1}{2 a_{1}}\right)
$$

and we use the superscript in $\mathbf{E}^{0}$ to emphasise that $H_{0}=B_{1}(0)=0$. Using the covariance of Brownian motion $\mathbf{E}\left(B_{s} B_{u}\right)=\min (s, u)$ and interchanging the order of integration, we get

$$
\begin{aligned}
& \mathbf{E}^{0}\left[\int_{0}^{t} \int_{0}^{t} f(s) f(u) H_{s} H_{u} d u d s\right] \\
= & 2 \int_{0}^{t} f(s) \int_{0}^{s} f(u) E\left(H_{s} H_{u}\right) d u d s \\
= & 2 \sigma_{1}^{2} \int_{0}^{t} f(s) e^{-a_{1} s} \int_{0}^{s} f(u) e^{-a_{1} u}\left(\frac{e^{2 a_{1} u}-1}{2 a_{1}}\right) d u d s .
\end{aligned}
$$

Putting $f \equiv 1$ in (2.3) gives the variance of $\int_{0}^{t} R_{s} d s$ to be

$$
\frac{\sigma_{1}^{2}}{a_{1}}\left(\frac{t}{a_{1}}+\frac{2 e^{-a_{1} t}}{a_{1}^{2}}-\frac{e^{-2 a_{1} t}}{2 a_{1}^{2}}-\frac{3}{2 a_{1}^{2}}\right) .
$$


At this point, it may be instructive to compare these results with the analogous ones for the AR(1) time series (1.9). The mean and variance of $\sum_{i=1}^{t} R_{i}$ has been obtained by Hürlimann (1992) and Wilkie (1995). Keeping to our notation established in (1.9), the mean of the accumulated force of inflation $\sum_{i=1}^{t} \tilde{R}_{i}$ is

$$
\mu t+\left(\tilde{R}_{0}-\mu\right) \frac{a\left(1-a^{t}\right)}{1-a}
$$

while in the continuous model the mean $\int_{0}^{t} R_{s} d s$ is (assuming $\phi=$ const.)

$$
\int_{0}^{t} \mu_{R}(s) d s=\mu t+\frac{R_{0}-\mu}{a_{1}}\left[1-e^{-a_{1} t}\right]
$$

where $\mu=\phi / a_{1}$ as before. We see immediately that (2.5) and (2.6) have the same form. To check that they in fact agree, recall that to obtain the timeseries (1.9) from (1.1), we discretized time into steps of size $h$. Therefore $\sum_{j=1}^{t / h} h \tilde{R}_{i}$ is precisely the Riemann-sum approximation to $\int_{0}^{t} R_{s} d s$. According to the formula (2.5), the mean of $\sum_{i=1}^{t / h} h R_{i}$ is

$$
\begin{aligned}
& \mu t+\left(\tilde{R}_{0}-\mu\right) h \frac{a\left(1-a^{t / h}\right)}{1-a} \\
& =\mu t+\left(R_{0}-\mu\right)\left(\frac{1-a_{1} h}{a_{1}}\right)\left[1-\left(1-a_{1} h\right)^{t / h}\right] \\
& \rightarrow \mu t+\frac{R_{0}-\mu}{a_{1}}\left[1-e^{-a_{1} t}\right]
\end{aligned}
$$

as $h \rightarrow 0$, which is precisely the mean of $\int_{0}^{t} R_{s} d s$ given by (2.6). Similarly, Hürlimann (1992) gives the variance of $\sum_{i=1}^{t} \widetilde{R}_{i}$ as

$$
\frac{\tilde{\sigma}_{1}^{2}}{(1-a)^{2}}\left[t-\frac{2 a\left(1-a^{t}\right)}{1-a}+\frac{a^{2}\left(1-a^{2 t}\right)}{1-a^{2}}\right]
$$

which has the same form as (2.4).

It is just as easy to obtain the distributions of the other processes in our model. Putting $f(s)=\rho e^{-\rho(t-s)}$ in $(2.3)$ we get that $\int_{0}^{t} e^{-\rho(t-s)} R_{s} d s$ has Gaussian distribution with mean

$$
\int_{0}^{t} \rho e^{-\rho(t-s)} \mu_{R}(s) d s
$$

and variance

$$
\frac{\rho \sigma_{1}^{2}}{a_{1}}\left(\frac{\left(a_{1}-\rho\right)^{2}-\left(a_{1}+\rho\right)\left(\rho e^{-2 a_{1} t}+a_{1} e^{-2 \rho t}\right)+4 a_{1} \rho e^{-\left(a_{1}+\rho\right) t}}{2\left(a_{1}-\rho\right)^{2}\left(a_{1}+\rho\right)}\right) .
$$


Putting $f=\beta+\gamma-\beta e^{-\lambda(t-s)}$ also gives an explicit expression for the variance of $\beta \int_{0}^{t}\left(1-e^{-\lambda(t-s)}\right) R_{s} d s+\gamma \int_{0}^{t} R_{s} d s$, although this is too messy to write down here - the formula is simplified somewhat by choosing $\gamma=0$ and simplified considerably by choosing $\gamma=-\beta$, for this would then reduce to $(2.7 \mathrm{~b})$. The full covariance structure of the process $t \mapsto \int_{0}^{t} f(s) R_{s} d s$ can also be obtained in this way.

Armed with these results, we can now state the distributions of interest. We have already found the distributions of $R_{t}$ and $P_{t}=\int_{0}^{t} R_{s} d s$ (see (2.2) and (2.4)). Applying the results (2.2) to the process $X$, we get from (1.3) and (1.4) that $\log Y_{t}$ has Gaussian distribution with mean

$$
\log Y_{*}+X_{0} e^{-a_{2} t}+b_{1}\left(\frac{1-e^{-a_{2} t}}{a_{2}}\right)+\zeta \mu_{R}(t)
$$

and variance

$$
\zeta^{2} v_{R}(t)+\sigma_{2}^{2}\left(\frac{1-e^{-2 a_{2} t}}{2 a_{2}}\right) .
$$

For the dividend index $D$, the result (2.3), with $f(s)=\beta+\gamma-\beta e^{-\lambda(t-s)}$, together with the analogous results (2.2) for $U$ give that $\log D_{t}$ has Gaussian distribution with mean

$$
\log D_{*}+b_{2} t+\int_{0}^{t}\left(\beta+\gamma-\beta e^{-\lambda(t-s)}\right) \mu_{R}(s) d s
$$

and variance

$$
\begin{aligned}
& \left(\eta_{2}^{2}+\eta_{3}^{2}\right) t \\
& \quad+2 \sigma_{1}^{2} \int_{0}^{t}\left(\beta+\gamma-\beta e^{-\lambda(t-s)}\right) e^{-a_{1} s} \int_{0}^{s}\left(\beta+\gamma-\beta e^{-\lambda(t-u)}\right)\left(\frac{e^{a_{1} u}-e^{-a_{1} u}}{2 a_{1}}\right) d u d s .
\end{aligned}
$$

Applying (2.2) to $V_{t}$ shows that it is Gaussian with mean

$$
\mu_{V}(t)=v e^{-a_{4} t}
$$

and variance

$$
v_{V}(t)=\sigma_{4}^{2}\left(\frac{1-e^{-2 a_{4} t}}{2 a_{4}}\right)
$$

The distribution of $C_{t}$ is the convolution of normal and $\log$ normal distributions and the results (2.2) and (2.7) show that $C_{t}$ has mean

$$
\xi \int_{0}^{t} \rho e^{-\rho(t-s)} \mu_{R}(s) d s+C_{*} e^{\mu_{\nu}(t)+v_{V}(t) / 2}
$$


and variance

$$
\begin{aligned}
& \frac{\rho \xi^{2} \sigma_{1}^{2}}{a_{1}}\left(\frac{\left(a_{1}-\rho\right)^{2}-\left(a_{1}+\rho\right)\left(\rho e^{-2 a_{1} t}+a_{1} e^{-2 \rho t}\right)+4 a_{1} \rho e^{-\left(a_{1}+\rho\right) t}}{2\left(a_{1}-\rho\right)^{2}\left(a_{1}+\rho\right)}\right) \\
& +C_{*}^{2} e^{2 \mu_{\nu}(t)+v_{v}(t)}\left(e^{v_{v}(t)}-1\right) .
\end{aligned}
$$

It is also possible to specify the full multivariate structure of $R, Y$ and $D$ using the methods here. Since $R, Y$ and $D$ are either Gaussian or log Gaussian, their joint law is specified once we have the covariances $\operatorname{Cov}\left(R_{t}, \log Y_{t}\right)$, $\operatorname{Cov}\left(R_{t}, \log D_{t}\right)$ and $\operatorname{Cov}\left(\log Y_{t}, \log D_{t}\right)$. For the most part, we only need to know the covariance stucture of the process $t \mapsto R_{t}$, which is given by

$$
\mathbb{E}\left[R_{t} R_{s}\right]=\mu_{R}(t) \mu_{R}(s)+\mathbb{E}\left[H_{s} H_{u}\right]=\mu_{R}(t) \mu_{R}(s)+\sigma_{1}^{2} e^{-a_{1} t}\left(\frac{e^{a_{1}, s}-1}{2 a_{1}}\right)
$$

if $s<t$. Thus, for example,

$$
\mathbb{E}\left[R_{t} \log D_{t}\right]=\mu_{R}(t)\left(\log D_{*}+b_{2} t\right)+\int_{0}^{t}\left(\beta+\gamma-\beta e^{-\lambda(t-s)}\right) \mathbb{E}\left[R_{t} R_{s}\right] d s
$$

and we can then substitute the relevant previous results into the above expression. In addition, we also need the covariance of $X_{t}$ and $Z_{2}(t)$, which is given by

$$
\mathbb{E}^{0}\left[X_{t} Z_{2}(t)\right]=\sigma_{2} e^{-a_{2} t} \min \left(\frac{e^{a_{2} t}-1}{2 a_{2}}, t\right)
$$

using the covariance of Brownian motion. The detailed computations of the covariances are left to the reader.

\section{EXPLICIT DISTRIBUTIONS IN THE DISCONTINUOUS CASE}

There have been some suggestions that Gaussian noise terms are not entirely appropriate for these models and that more realistically, the noise should have jumps. In this section, we perform the same analysis as in Section 2 on the assumption that the $Z_{i}$ are symmetric pure-jump Lévy processes.

From the analysis in Section 2, it is clear that once we know what the law of $\int_{0}^{t} f(s) Z_{s} d s$ is for fixed $t$ (where $f(s)$ or $f(t, s)$ is a suitable function and $Z$ is a generic Lévy process), we can obtain the necessary explicit distributions. It all turns out to rest on the following simple lemma allowing the interchange of order of integration:

LEMMA 3.1: Let $f$ and $g$ be Riemann-integrable functions. Then the laws of

$$
\int_{0}^{t} f(s) \int_{0}^{s} g(u) d Z_{u} d s \text { and } \int_{0}^{t} g(u) \int_{u}^{t} f(s) d s d Z_{u}
$$


are the same for each fixed $t$ and the common law is given by

$$
\mathbb{E}\left[\exp \left\{-i \theta \int_{0}^{t} g(u) \int_{u}^{t} f(s) d s d Z_{u}\right\}\right]=\exp \left\{-\int_{0}^{t} \psi(\theta g(u)[F(t)-F(u)]) d u\right\}
$$

where $\psi$ is given by the Lévy-Khintchine formula (1.10) and $F(u)=\int_{0}^{u} f(s) d s$.

The proof, although not very pretty, uses only well-known standard results in the theory of stochastic integration and Lévy processes and is presented in the Appendix.

\section{Remarks:}

(i) The above lemma is trivial if $Z$ has finite variation, for then the integral $\int_{0}^{f} g(s) d Z_{s}$ exists as an ordinary Riemann-Stieltjes integral. Changing the order of integration as for ordinary integrals, we actually have the much stronger result that

$$
\mathbb{P}\left(\int_{0}^{t} f(s) \int_{0}^{s} g(u) d Z_{u} d s=\int_{0}^{t} g(u) \int_{u}^{t} f(s) d s d Z_{u} \quad \forall t\right)=1
$$

When $Z$ has infinite variation, the integral with respect to $Z$ is a "genuine" stochastic integral. In this case, we have to emphasise that Lemma 3.1 holds only for fixed $t$; the two integrals clearly cannot have the same law as processes since the former is a process of finite variation while the latter has infinite variation.

(ii) Since $t$ is a fixed parameter in the present context, Lemma 3.1 holds equally if we allow $f$ and $g$ to also depend on $t$, which we need to do for some of the processes considered earlier.

(iii) Note that a simple special case of (3.1) is that

$$
\mathbb{E}\left[\exp \left\{-i \theta \int_{0}^{t} G(t, u) d Z_{u}\right\}\right]=\exp \left\{-\int_{0}^{t} \psi(\theta G(t, u)) d u\right\}
$$

for any (Riemann-integrable) function $G$.

Consider now the model described in Section 1 where the $Z_{i}$ are symmetric Lévy processes with jumps. From the explicit formula (1.2) for $R$, we see that to find the law of $R_{t}$ we can apply (3.2) with $G(t, u)=\sigma_{l} e^{-a_{1}(t-u)}$, in which case we obtain

$$
\mathbb{E}\left[e^{-i \theta R_{t}}\right]=\exp \left\{-i \theta \mu_{R}(t)-\int_{0}^{t} \psi\left(\theta \sigma_{1} e^{-a_{l}(t-u)}\right) d u\right\},
$$

where $\mu_{R}(t)$ is as defined by (2.2a). In a similar way we can obtain the laws of the processes $X, U$ and $V$ introduced in Section 1. For the law of $\int_{0}^{t} R_{s} d s$, we can apply Lemma 3.1 with $g(t, u)=\sigma_{1} e^{-a_{1}(t-u)}, f \equiv 1$ and for the law of $\int_{0}^{t} \rho e^{-\rho(t-s)} R_{s} d s$ we can take $g(t, u)=\sigma_{1} e^{-a_{1}(t-u)}, f(t, s)=\rho e^{-\rho(t-s)}$. In this 
way, we obtain the distributions of $R_{t}, S_{t}, C_{t}$ and $\log D_{t}$ in a similar manner to Section 2. However, the joint distribution is much more difficult to obtain.

We end this section with a brief word on some specific examples of Lévy processes one might choose to use in these models. We just mention two commonly used Lévy processes. One is the symmetric $\alpha$-stable process, whose Lévy exponent is $\psi(\theta)=|\theta|^{\alpha}$ and whose Lévy measure is

$$
\nu(d x)=\frac{C_{\alpha}}{|x|^{1+\alpha}} d x, \quad x \neq 0,
$$

where $C_{\alpha}=\pi^{-1} \Gamma(1+\alpha) \sin (\pi \alpha / 2)$. (Here $0<\alpha<2 ; \alpha=2$ corresponds to the Gaussian distribution and $\alpha=1$ gives the Cauchy distribution). Stable distributions are examples of so-called heavy-tailed distributions. One of the disadvantages of stable processes is that they do not have higher order moments than 1 (for $\alpha \leq 1$ they do not even have a first moment) which may cause awkward problems, for example, when we take exponentials of stable processes as we are frequently doing in these models.

Another commonly used class of Lévy processes which overcomes this problem is the Gamma process. A Lévy process $Y$ is said to be Gamma with parameters $\left(\alpha, \beta\right.$ where $\alpha, \beta>0$ if $\mathbb{P}\left(Y_{1} \leq x\right)=\Gamma(\alpha)^{-1} \beta^{\alpha} \int_{0}^{x} y^{\alpha-1} e^{-\beta y} d y$. Hence

$$
\mathbb{E}\left[e^{-i \theta Y_{t}}\right]=\left(\frac{\beta}{\beta+i \theta}\right)^{\alpha t}=\exp \left\{-\alpha t \log \left(1+\frac{i \theta}{\beta}\right)\right\} .
$$

Note that such a process is non-decreasing, so to obtain a symmetric process, we simply take two independent copies $Y$ and $\tilde{Y}$ and define $Z=Y-\tilde{Y}$. The process $Z$ is therefore a symmetric Lévy process with Lévy exponent $\psi(\theta)=\alpha \log \left(1+\theta^{2} / \beta^{2}\right)$ and Lévy measure $\nu(d x)=\alpha|x|^{-1} e^{-\beta|x|} d x$. Looking at the Lévy decomposition, since $\int_{\{|x|<1\}}|x| \nu(d x)<\infty$, we see that $Z$ has finite variation and since $\int_{\{|x| \geq 1\}}|x|^{n} \nu(d x)<\infty, Z_{t}$ has finite moments of all orders.

Applying Lemma 3.1 we obtain (replacing $i \theta$ with $\theta$ for convenience)

$$
\mathbb{E}\left[\exp \left\{-\theta \int_{0}^{t} f(t-s) d Z_{s}\right\}\right]=\exp \left\{-\alpha \int_{0}^{t} \log \left(1-\frac{\theta^{2} f(t-s)^{2}}{\beta^{2}}\right) d s\right\}
$$

and

$$
\begin{aligned}
& \mathbb{E}\left[\exp \left\{-\theta \int_{0}^{t} g(u) \int_{u}^{t} f(s) d s d Z_{u}\right\}\right] \\
& =\exp \left\{-\alpha \int_{0}^{t} \log \left(1-\frac{\theta^{2} g(u)^{2}}{\beta^{2}}\left[\int_{u}^{t} f(s) d s\right]^{2}\right) d u\right\} .
\end{aligned}
$$

Although in general it is not possible to give explicit formulae for the integrals in (3.3) and (3.4) for our choices of $f$ and $g$ as in the preceding two sections, the Laplace transforms (3.3-4) do give relatively simple expressions for the moments, involving integrals which can be readily evaluated by numerical means. 


\section{CONCLUDING REMARKS}

We have concentrated here on obtaining explicit formulae, both in the case where our SDEs are driven by Brownian motions and in the case where they are driven by symmetric Lévy processes with jumps. Of course, many other questions - which we have not considered - do not admit explicit answers and one must then resort to numerical solutions. It is not our intention here to give a detailed quantitative analysis of numerical simulations of the models presented in the preceding sections, as this could well constitute a paper in its own right. We simply present some examples of numerical simulations to give a feel for what these processes look like. In the case of SDEs driven by Brownian motion, great advances have been made in recent years in numerical methods for solving them. For a comprehensive survey of these techniques as well as an extensive bibliography on the subject, we refer the reader to Kloeden and Platen (1992). By contrast, numerical methods for SDEs driven by processes with jumps, such as stable processes, have received far less attention until recently and the literature on this subject is more limited: a systematic treatment in book form can be found in Janicki and Weron (1993).

For simplicity, we present some simulations for the inflation process $R_{t}$ only since of the four components, this is closest to the time-series model of Wilkie. Figure 1 shows three trajectories of the process $R_{t}$, in the case where the noise $Z_{i}$ is Brownian motion. The scaling used is such that the time interval $[0,1]$ corresponds to a period of 50 years. Specifically, in the context of Remark (ii) in Section 1, we have used $h=0.02$ and in equation (1.2) our choice of $\phi$ is $\phi(t)=b+c \cos (2 \pi t / h)$. Since the picture is only intended to give a qualitative indication of how the process behaves, the actual numerical values on the vertical axes are not of any great importance: the parameter values in Wilkie (1986) are used as a rough guide to the sort of values which might be appropriate for the parameters here - in particular, the parameter values of Wilkie are rescaled in the manner discussed in Remark (ii) of Section 1.

Throughout, we have taken the various parameters in our models as given quantities and we have said nothing abcut the problems of their estimation. There is some discussion of this question in $\$ 6.4$ and $\$ 13.2$ of Kloeden and Platen (1992) which is especially relevant to the linear equations which appear repeatedly in our models. 


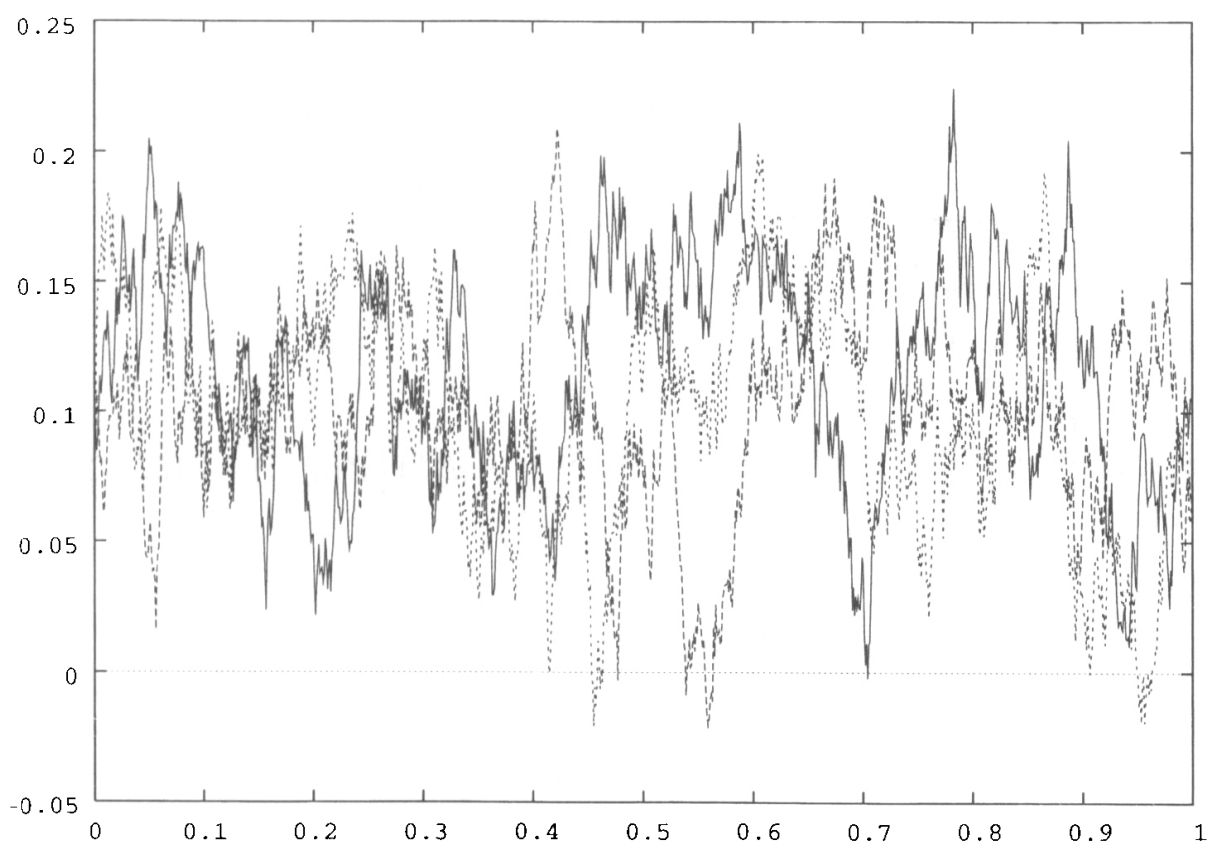

Figurf 1: SAMPle PATH REALIZATIONS OF THE FORCE OF INFLATION PROCESS $R_{t}$.

\section{APPENDIX: ProOF OF LEMMA 3.1}

Consider first the integral $I(s):=\int_{0}^{s} g(u) d Z_{u}$. Take a sequence of partitions $\left(u_{k}^{(n)}, u_{k+1}^{(n)}\right]$ of the interval $[0, t]$, such that $\sup _{k}\left|u_{k+1}^{(n)}-u_{k}^{(n)}\right| \rightarrow 0$ as $n \rightarrow \infty$. It is known that, as $n \rightarrow \infty$,

$$
I_{n}(s):=\sum_{u_{k}^{(n)} \leq s} g\left(u_{k}^{(n)}\right)\left(Z\left(u_{k+1}^{(n)}\right)-Z\left(u_{k}^{(n)}\right)\right) \rightarrow \int_{0}^{s} g(u) d Z_{u}
$$

in probability uniformly in $s$ over the time interval $[0, t]$ (see Protter (1990)). Therefore, there is a subsequence $\left(n_{i}\right)$ such that $I_{n_{i}}(s) \rightarrow I(s)$ almost surely as $i \rightarrow \infty$ and without loss of generality we can assume that $I_{n}(s) \rightarrow I(s)$ almost surely. Next, take a different sequence of successively refining partitions of $[0, t]$ and call this $\left[s_{j}^{(m)}, s_{j+1}^{(m)}\right)$. Put

$$
F_{m}\left(u_{k}^{(n)}, t\right)=\sum_{s_{j}^{(m)} \geq u_{k}^{(n)}} f\left(s_{j}^{(m)}\right)\left(s_{j+1}^{(m)}-s_{j}^{(m)}\right) .
$$


(Of course, $F_{m}\left(u_{k}^{(n)}, t\right) \rightarrow F(t)-F\left(u_{k}^{(n)}\right)$ as $n \rightarrow \infty$.). We then have

$$
\begin{aligned}
& \lim _{m \rightarrow \infty} \lim _{n \rightarrow \infty} \sum_{s_{j}^{(m)} \leq t} f\left(s_{j}^{(m)}\right)\left(s_{j+1}^{(m)}-s_{j}^{(m)}\right) \sum_{\substack{(n) \leq s_{j}^{(m)} \\
u_{k}}} g\left(u_{k}^{(n)}\right)\left(Z\left(u_{k+1}^{(n)}\right)-Z\left(u_{k}^{(n)}\right)\right) \\
& =\int_{0}^{t} f(s) \int_{0}^{s} g(u) d Z_{u} d s
\end{aligned}
$$

and so for fixed $n$,

$$
\begin{aligned}
& \mathbb{E}\left[\exp \left\{-i \theta \sum_{s_{j}^{(m)} \leq t} f\left(s_{j}^{(m)}\right)\left(s_{j+1}^{(m)}-s_{j}^{(m)}\right) \sum_{u_{k}^{(n)} \leq s_{j}^{(m)}} g\left(u_{k}^{(n)}\right)\left(Z\left(u_{k+1}^{(n)}\right)-Z\left(u_{k}^{(n)}\right)\right)\right\}\right] \\
& =\mathbb{E}\left[\exp \left\{-i \theta \sum_{u_{k}^{(n)} \leq t} g\left(u_{k}^{(n)}\right)\left(Z\left(u_{k+1}^{(n)}\right)-Z\left(u_{k}^{(n)}\right)\right) \sum_{s_{j}^{(m)} \geq u_{k}^{(n)}} f\left(s_{j}^{(m)}\right)\left(s_{j+1}^{(m)}-s_{j}^{(m)}\right)\right\}\right] \\
& =\prod_{u_{k}^{(n)} \leq t} \exp \left\{-\left(u_{k+1}^{(n)}-u_{k}^{(n)}\right) \psi\left[\theta g\left(u_{k}^{(n)}\right) F_{m}\left(u_{k}^{(n)}, t\right)\right]\right\} \\
& =\exp \left\{-\sum_{u_{k}^{(n)} \leq t}\left(u_{k+1}^{(n)}-u_{k}^{(n)}\right) \psi\left[\theta g\left(u_{k}^{(n)}\right) F_{m}\left(u_{k}^{(n)}, t\right)\right]\right\} \\
& \rightarrow \exp \left\{-\sum_{u_{k}^{(n)} \leq t}\left(u_{k+1}^{(n)}-u_{k}^{(n)}\right) \psi\left[\theta g\left(u_{k}^{(n)}\right)\left(F(t)-F\left(u_{k}^{(n)}\right)\right)\right]\right\}
\end{aligned}
$$

as $m \rightarrow \infty$. In the above calculation, we have used the stationary independent increments property of $Z$ and the fact that $\mathbf{E}\left[e^{-i \theta\left(Z_{t}-Z_{s}\right)}\right]=e^{-(t-s) \psi(\theta)}$. Letting $n \rightarrow \infty$ in (A1) then gives the right-hand side of (3.1).

For the integral $\int_{0}^{t} g(u) \int_{u}^{t} f(s) d s d Z_{u}$, we know that

$$
\sum_{k} g\left(u_{k}^{(n)}\right)\left(F(t)-F\left(u_{k}^{(n)}\right)\right)\left(Z\left(u_{k+1}^{(n)}-Z\left(u_{k}^{(n)}\right)\right) \rightarrow \int_{0}^{t} g(u) \int_{u}^{t} f(s) d s d Z_{u}\right.
$$

almost surely as $n \rightarrow \infty$ (passing to a subsequence if necessary). A similar calculation as in (A1) easily yields the identity (3.1).

\section{ACKNOWLEDGEMENT}

I am grateful to David Wilkie for his many detailed and helpful comments on an earlier draft of this paper. 


\section{REFERENCES}

HÜRlimanN, W. (1992), "Numerical evaluation of the Wilkie inflation model", Insurance: Mathematics \& Economics 11, 311-314.

JANicki, A. and Weron, A. (1993), Simulation and Chaotic Behaviour of $\alpha$-Stable Stochastic Processes, Marcel Dekker.

Kloeden, P.E. and Platen, E. (1992), Numerical Solution of Stochastic Differential Equations, Springer.

PROTTER, P. (1990), Stochastic Integration and Differential Equations, Springer.

Rogers, L.C.G. and Williams, D. (1987), Diffusions, Markov Processes and Martingales, Vol. 2: Itô Calculus, Wiley.

WILKIE, A.D. (1986), "A stochastic investment model for actuarial use", Trans. Faculty of Actuaries 39, 341-373.

Wilkie, A.D. (1995), "More on a stochastic investment model for actuarial use", British Actuarial Journal 1, 777-964.

\section{TERENCE CHAN}

Dept. of Actuarial Mathematics

and Statistics

Heriot-Watt University

Edinburgh EH $144 A S$ U.K. 